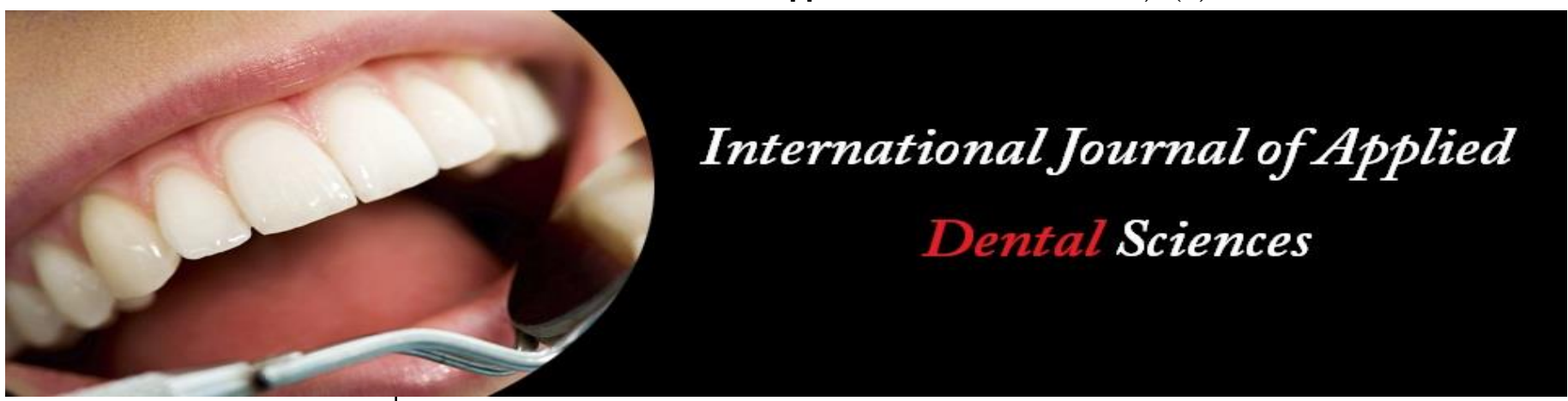

ISSN Print: 2394-7489

ISSN Online: 2394-7497

IJADS 2021; 7(3): 369-373

(C) 2021 IJADS

www.oraljournal.com

Received: 04-05-2021

Accepted: 06-06-2021

Zenaida Carolina Garcia Castañeda Master in Sciences Student, Universidad Autonoma de Nuevo Leon, Facultad de Odontologia, Monterrey, Nuevo Leon, CP. Mexico

Hugo Villarreal Garza Associate Professor, Universidad Autonoma de Nuevo Leon, Facultad de Odontologia, Monterrey, Nuevo Leon, CP, Mexico

Hugo Felix Madla Alanis Professor, Universidad Autonoma de Nuevo Leon, Facultad de Odontologia, Monterrey, Nuevo Leon, CP, Mexico

Aurora Lucero Reyes Professor, Universidad Autonoma de Tlaxcala, Facultad de Odontologia, Tlaxcala, Mexico

Patricia Limon Huitron Professor, Universidad Autonoma de Tlaxcala, Facultad de Odontologia, Tlaxcala, Mexico

Rosa Isela Sanchez-Najera Professor, Universidad Autonoma de Nuevo Leon, Facultad de Odontologia, Monterrey, Nuevo Leon, CP, Mexico

Juan Manuel Solis-Soto Professor, Universidad Autonoma de Nuevo Leon, Facultad de Odontologia, Monterrey, Nuevo Leon, CP, Mexico
Corresponding Author:

Zenaida Carolina Garcia Castañeda Master in Sciences Student, Universidad Autonoma de Nuevo Leon, Facultad de Odontologia, Monterrey, Nuevo Leon, CP, Mexico

\section{Microorganisms in persistent apical periodontitis: A review}

\section{Zenaida Carolina Garcia Castañeda, Hugo Villarreal Garza, Hugo Felix Madla Alanis, Aurora Lucero Reyes, Patricia Limon Huitron, Rosa Isela Sanchez-Najera and Juan Manuel Solis-Soto}

\section{DOI: https://doi.org/10.22271/oral.2021.v7.i3f.1323}

\section{Abstract}

Introduction: Apical periodontitis is a sequela of endodontic infection, which manifests as a host defense response to the microbial challenge emanating from the root canals. To achieve an optimal outcome, microorganisms must be eliminated or reduced to levels that allow healing of the periradicular tissue.

Objective: To analyze the literature on microorganisms, such as Enterococcus faecalis, Fusobacterium nucleatum, Candida albicans, Epstein-Barr, which are important in persistent apical periodontitis.

Methodology: Articles on the subject published through PubMed, SCOPUS and Google Scholar databases were analyzed, with emphasis on the last 5 years. It was performed with the words "Enterococcus faecalis", "Fusobacterium nucleatum", "Candida albicans", "Epstein-Barr", "Herpesviridae", "Root canals", "Persistent apical periodontitis".

Results: E. faecalis involved in persistent apical periodontitis because of its adaptability to extreme environments, growing in alkaline $\mathrm{pH}$ and using periodontal ligament fluids as nutrients. Lysed Fusobacterium nucleatum cells could potentially increase the severity of persistent apical periodontitis. Candida albicans is one of the dominant pathogens in persistent apical periodontitis because of its membrane protein Msb2. Epistein-Barr virus may be implicated in the pathogenesis of apical periodontitis by direct cytopathic action on infected cells, however, its replication in persistent apical periodontitis is still unclear.

Conclusions: The microbiota of teeth with persistent apical periodontitis, presents a mixed and complex profile, it is important to know the role of these microorganisms, because microbial persistence, seems to be the most important factor in root canal treatment failure.

Keywords: Enterococcus faecalis, Fusobacterium nucleatum, Candida albicans, epstein-barr, herpesviridae, root canals, apical periodontitis

\section{Introduction}

For a better prognosis of root canal system treatment, an adequate knowledge of the microbial flora of the root, especially the apical portion, is necessary ${ }^{[1]}$. Apical periodontitis is an inflammatory disease of the periradicular tissues caused by bacteria colonizing necrotic root canals ${ }^{[2]}$. Persistent apical periodontitis is a situation involving an inflammatory and immune response caused mainly by an anaerobic polymicrobial infection of the root canal system ${ }^{[3]}$. Microorganisms are considered to play the main etiological role in the formation of endodontic diseases ${ }^{[4]}$. The endodontic microbiota is composed of a subset of microbiota present in the oral cavity, consisting of predominantly anaerobic bacterial species, some fungal species and viruses ${ }^{[5]}$. Microbial factors in necrotic root canals (e.g., Endotoxin) can spread to the apical tissue, causing and supporting a chronic inflammatory burden. Thus, apical periodontitis is the result of the complex interplay between microbial factors and host defense against invasion of periradicular tissues ${ }^{[6]}$. The complexity and variability of the root canal system, along with the multi-species nature of biofilms, make disinfection of this system extremely challenging ${ }^{[7]}$. Understanding the formation and progression of apical periodontitis can help increase knowledge of pathogenic mechanisms, improve diagnosis, and provide support for different therapeutic strategies ${ }^{[8]}$. 
It is of great importance to recognize the different microorganisms found in the root canal system with persistent apical periodontitis in order to successfully perform conventional endodontic retreatments and thus reduce periradicular surgeries. Therefore, the aim of this article is to analyze the literature about microorganisms, such as Enterococcus faecalis, Fusobacterium nucleatum, Candida albicans, Epstein-Barr, which are important in persistent apical periodontitis, particularly their characteristics, biofilm, virulence factors, survival mechanisms, as well as studies that prove this relationship.

\section{Materials and Methods}

Articles on the subject published through the PubMed, SCOPUS and Google Scholar databases were analyzed, with emphasis on the last 5 years. The quality of the articles was evaluated using PRISMA guidelines, i.e., identification, review, choice and inclusion. The quality of the reviews was assessed using the measurement tool for evaluating systematic reviews (AMSTAR-2) ${ }^{[9]}$. The search was performed using Boolean logical operators AND, OR and NOT. It was realized with the words "Enterococcus faecalis", "Candida albicans", "Herpesviridae", "Epstein-Barr", "Fusobacterium nucleatum", "Root canals", "Persistent apical periodontitis".

\section{Results \& Discussion \\ 3.1 Enterococcus faecalis}

\subsubsection{Characteristics}

Enterococcus faecalis is an aerotolerant gram-positive bacterium that is widely distributed in the natural environment. The most important characteristics of E. faecalis are its high adaptability in adverse environmental conditions and its potential development of antibiotic resistance ${ }^{[10]}$. It is the most frequent species present in post-treatment disease and plays an important role in persistent periapical infections [11].

\subsubsection{Biofilm}

Can form biofilms with a hard extracellular polymeric polymeric matrix; this biofilm can serve as a protective barrier against antibacterial agents ${ }^{[12]}$. It confers phenotypic antimicrobial tolerance to biofilm-associated bacteria ${ }^{[13]}$.

\subsubsection{Virulence factors.}

The virulence factors of $E$. faecalis include bile salt hydrolase, cytolysin toxin, capsular polysaccharides, gelatinase, lipoproteins and other surface-associated LPxTG aggregating substances ${ }^{[14]}$. E. faecalis can establish extra-root infection by secreting toxins directly through inducing inflammation indirectly, as well as can gain and transfer extra chromosomal elements and coding virulence traits, which help to colonize and compete with other bacteria ${ }^{[15]}$. In addition, they can protect bacteria from immune detection or phagocytosis, and serve as an effective immune evasion mechanism ${ }^{[16]}$.

\subsubsection{Survival mechanisms.}

It has an adaptability in root canals due to its abilities to grow with or without oxygen, grow at alkaline $\mathrm{pH}$, survive at temperatures between 10-60 degrees Celsius. To these survival mechanisms, we can also add the ability of $E$. faecalis to live without nutrients, can survive in the presence of intra-canals drugs, survive high salinity, to acquire resistance to antibiotics, in particular erythromycin and azithromycin, to invade dentinal tubules, to use periodontal ligament fluids as nutrients and to adhere to collagen ${ }^{[17]}$. It remains viable and proliferate in treated root canals for a long period of time ${ }^{[18]}$.

\subsubsection{Studies of Enterococcus faecalis showing relationship with persistent apical periodontitis}

E. faecalis is present in the root canals of patients with persistent periapical periodontitis and is believed to be involved in the persistence of periapical lesions due to the difficulty in adequately debriding root canals during root canal preparation and dressing ${ }^{[19]}$. Enterococcus faecalis is not among the leading causes of primary endodontic infections, but causes one of the most recurrent and persistent forms of chronic apical periodontitis ${ }^{[20]}$.

Enterococcus faecalis is an aerotolerant gram-positive bacterium, which can form biofilms with a hard extracellular polymeric extracellular matrix, as well as gain and transfer extra chromosomal elements and coding virulence traits In fact, this bacterium can enter a viable state, which consists of an adaptive mechanism when exposed to unfavorable conditions. E. faecalis is present in the root canals of patients with persistent periapical periodontitis and is considered to be the bacterium most related to endodontic treatment failures due to its high adaptability and potential development of antibiotic resistance.

\subsection{Fusobacterium nucleatum}

\subsubsection{Characteristics}

It is a gram-negative anaerobic oral commensal ${ }^{[21]}$. Among its characteristics we can find that it has properties that make it able to escape disinfection measures, ability to form a biofilm, to locate in areas unreachable for root canal instrumentation techniques, synergism and the ability to express survival genes ${ }^{[22]}$.

\subsubsection{Biofilm}

Biofilm formation by $F$. nucleatum can provide protection to cells when exposed to alkaline environments. Bacteria growing in biofilms exhibit altered phenotypes and are more resistant to antimicrobial agents and the host immune system. ${ }^{[23]}$. In one study, $P$. gingivalis was shown to enhance biofilm formation by $F$. nucleatum by releasing diffusible signaling molecules (autoinducer-2-producing gene luxS) ${ }^{[24]}$.

\subsubsection{Virulence factors}

Possess virulence factors that allow them to survive in hostile environments by selectively modulating the host immunoinflammatory response. ${ }^{[25]}$. Additional virulence properties associated with $F$. nucleatum include hemolytic activity and the ability to produce hydrogen sulfide. $F$. nucleatum is well known for its invasive properties, which may allow it to enter the bloodstream, migrate and cause infections in other parts of the body ${ }^{[26]}$. Its pathogenicity depends on the degree of anaerobiosis, $\mathrm{pH}$ level, availability of exogenous and endogenous nutrients ${ }^{[27]}$.

\subsubsection{Survival mechanisms}

Can survive and multiply despite death during root canal treatment, lysed cells present in the dentinal tubule or biofilm can act as plasmid or chromosomal DNA donors. Plasmids or smaller peptides called pheromones can impart drug resistance and virulence to other microbes such as Enterococcus faecalis, thereby increasing the pathogenicity of other microorganisms ${ }^{[28]}$. It also produces poly-gammaglutamate, which has a role in virulence and survival under 
some unfavorable conditions ${ }^{[29]}$.

\subsubsection{Studies of Fusobacterium nucleatum showing} relationship with persistent apical periodontitis.

Fusobacterium nucleatum was found to play a determinant role in the pathogenicity of primary endodontic infections ${ }^{[30]}$. It is very commonly found in bacteriological profile evaluations in the apical root segment of patients with primary apical periodontitis ${ }^{[31]}$ and potentially increase the severity of persistent apical periodontitis ${ }^{[32]}$.

Fusobacterium nucleatum is a gram-negative anaerobic oral commensal, biofilm-forming oral commensal that can provide protection to cells when exposed to alkaline environments, possess virulence factors that allow them to survive in hostile environments, It is very commonly found in apical root segment of patients with primary apical periodontitis, in turn could potentially increase the severity of persistent apical periodontitis.

\subsection{Candida albicans}

\subsubsection{Characteristics}

It is a common member of the human microflora and is an important human opportunistic fungal pathogen [33]. Characteristics of $C$. albicans include the ability to monoinfect, survival in nutrient-poor environments, bacterial coaggregation, dimorphism, adaptation to variable environmental conditions, tissue adherence, production of hydrolytic enzymes, biofilm formation, and modulation of the host immune response ${ }^{[34]}$.

\subsubsection{Biofilm}

C. albicans biofilms are inherently resistant to antifungal drugs, the host immune system and environmental stresses [35]. C. albicans in particular, can coexist with multiple bacterial species and are known for their ability to form biofilms with them ${ }^{[36]}$. One study indicated that different phenotypes of $C$. albicans biofilms cultured on a noncomplex surface topography have the potential to differentially tolerate standard endodontic irrigation protocols [37]. As there is also the potential for biofilm interactions between kingdoms, bacteria and yeasts in the root canal, which are likely to complicate infection and require alternative treatment strategies ${ }^{[38]}$.

\subsubsection{Virulence factors.}

An important virulence attribute is its ability to form biofilms, densely clustered communities of cells attached to a surface [39]. The C. albicans membrane protein Msb2 is able to bind and inactivate host defense proteins and antibiotics, such as daptomycin. Production of Msb2 could subsequently provide the same protection to E. faecalis, leading to long-term colonization of the root canal ${ }^{[40]}$. Candida could facilitate the rise of pathogenic microorganisms as it modifies the host defense mechanism ${ }^{[41]}$.

\subsubsection{Survival mechanisms}

It binds to tooth dentin, forms biofilms and invades dentinal tubules to resist intracanal disinfectants and endodontic treatments ${ }^{[42]}$. It has the ability to form bilayer biofilm, rich in an extracellular matrix composed of carbohydrates, proteins, phosphorus and hexosamines, which allows good tolerance and growth in nutrient-restricted environments, such as occurs in retreatment of the canal system. In addition, it has been considered tolerant to chemical compounds commonly used in biomechanical instrumentation of infected roots, canals or dressings, such as calcium hydroxide ${ }^{[43]}$.

\subsubsection{Candida albicans studies showing relationship with persistent apical periodontitis}

It has been demonstrated the Prevalence of Candida albicans in primary endodontic infections associated with a higher frequency of apical periodontitis in patients with type 2 diabetes mellitus, It is one of the dominant pathogens in periapical lesions associated with persistent apical periodontitis ${ }^{[44,45]}$.

Candida albicans is a human opportunistic fungal pathogen, whose important virulence attribute is its ability to form biofilms which are intrinsically resistant to antifungal drugs, the host immune system and environmental stresses. With a correlation in immunosuppressed patients, being one of the dominant pathogens associated with persistent apical periodontitis.

\subsection{Epstein-Barr}

\subsubsection{Characteristics}

Epstein-Barr virus (EBV), a gamma-herpesvirus, latently infects more than $90 \%$ of adult humans worldwide ${ }^{[46]}$. Once Epistein-Barr Virus infects a human being, it can never be eliminated despite antiviral therapy ${ }^{[47]}$. It is the virus most associated with endodontic disease ${ }^{[48]}$. The herpesviridae correspond to a DNA virus (linear double helix), with a virion size varying between 120 and $150 \mathrm{~nm}$. The herpesviridae have an icosahedral capsid, a proteinaceous tegument and a sheath with viral glycoproteins ${ }^{[49]}$.

\subsubsection{Virulence factors}

Herpesviruses have evolved several indirect mechanisms, for example; inhibition of major histocompatibility complex class I and II expression on the surface of macrophages, induction of proinflammatory cytokine production, evasion of apoptosis, among others, which impair local host defense and increase the aggressiveness of bacterial pathogens resident at the site of inflammation [50]. EBV infection induces the expression of proinflammatory cytokines such as tumor necrosis factor $\alpha$, interleukin (IL) $-1 \beta$, IL-8, IL-10, IL-12 and IL-17 ${ }^{[51]}$.

\subsubsection{Survival mechanisms}

EBV has evasion strategies that it employs to facilitate immune escape during latency ${ }^{[52]}$. High levels of inflammatory cells that have latent herpesvirus, in combination with a compromised host response in periapical lesions, may create favorable conditions for reactivation ${ }^{[53]}$.

\subsubsection{Epstein-Barr studies showing association with persistent apical periodontitis.}

It has been frequently detected in apical periodontitis and associated with large lesions and root cysts, both in immunocompetent and immunocompromised patients. It may be implicated in the pathogenesis of apical periodontitis either by direct cytopathic action on infected cells or by virusinduced impairment of host defense, which in turn aids bacterial growth ${ }^{[54]}$. However, replication of Epistein-Barr Virus in persistent apical periodontitis has not yet been elucidated ${ }^{[55]}$.

Epstein-Barr virus (EBV), a gamma-herpesvirus, latently infects more than $90 \%$ of adult humans worldwide, is most associated with endodontic disease. Epstein-Barr virus has been detected in apical periodontitis, large lesions and root cysts. The presence of virus may cause local 
immunosuppression, which favors bacterial growth in the periapical. The replication of Epistein-Barr Virus in persistent apical periodontitis has not yet been elucidated and further studies are needed to verify this.

\section{Conclusions}

E. faecalis is thought to be involved in the persistence of periapical lesions due to its abilities to adapt to extreme environments, grow at alkaline $\mathrm{pH}$, and use periodontal ligament fluids as nutrients. Lysed Fusobacterium nucleatum cells could potentially increase the severity of persistent apical periodontitis as they act as chromosomal DNA donors that generate resistance to other microorganisms. Candida albicans is one of the dominant pathogens in persistent apical periodontitis because of its membrane protein Msb2 that is able to bind and inactivate host defense proteins and antibiotics. Epistein-Barr virus may be implicated in the pathogenesis of apical periodontitis by direct cytopathic action on infected cells, however, the replication of EpisteinBarr virus in persistent apical periodontitis is still unclear.

\section{References}

1. Tatikonda A, Sudheep N, Biswas KP, Gowtham K, Pujari S, Singh P. Evaluation of Bacteriological Profile in the Apical Root Segment of the Patients with Primary Apical Periodontitis. J Contemp Dent Pract 2017;18(1):44-48

2. Bouillaguet S, Manoil D, Girard M, Louis J, Gaïa N, Leo $\mathrm{S}$, et al. Root Microbiota in Primary and Secondary Apical Periodontitis. Front Microbiol 2018;9:2374.

3. Mazzi-Chaves JF, Petean IBF, Soares IMV, Salles AG, Antunes LAA, Segato RAB, et al. Influence of Genetic Polymorphisms in Genes of Bone Remodeling and Angiogenesis Process in The Apical Periodontitis. Braz Dent J 2018;29(2):179-183.

4. Liu D, Peng X, Wang S, Han Q, Li B, Zhou X, et al. A novel antibacterial resin-based root canal sealer modified by Dimethylaminododecyl Methacrylate. Sci Rep 2019;9(1):10632.

5. Aw V. Discuss the role of microorganisms in the etiology and pathogenesis of periapical disease. Aust Endod $\mathbf{J}$ 2016;42(2):53-9.

6. Gomes BPFA, Herrera DR. Etiologic role of root canal infection in apical periodontitis and its relationship with clinical symptomatology. Braz Oral Res 2018;32(suppl 1):e69.

7. Neelakantan P, Romero M, Vera J, Daood U, Khan AU, Yan A, et al. Biofilms in Endodontics-Current Status and Future Directions. Int J Mol Sci 2017;18(8):1748.

8. Braz-Silva PH, Bergamini ML, Mardegan AP, De Rosa CS, Hasseus B, Jonasson P. Inflammatory profile of chronic apical periodontitis: a literature review. Acta Odontol Scand 2019;77(3):173-180.

9. Shea BJ, Reeves BC, Wells G, Thuku M, Hamel C, Moran J, et al. AMSTAR 2: a critical appraisal tool for systematic reviews that include randomised or nonrandomised studies of healthcare interventions, or both. BMJ 2017 21;358:j4008.

10. Ali L, Goraya MU, Arafat Y, Ajmal M, Chen JL, Yu D. Molecular Mechanism of Quorum-Sensing in Enterococcus faecalis: Its Role in Virulence and Therapeutic Approaches. Int J Mol Sci 2017;18(5):960.

11. Zilm PS, Butnejski V, Rossi-Fedele G, Kidd SP, Edwards S, Vasilev K. D-amino acids reduce Enterococcus faecalis biofilms in vitro and in the presence of antimicrobials used for root canal treatment. PLoS One.
2017;12(2): e0170670.

12. Chiniforush N, Pourhajibagher M, Shahabi S, Kosarieh E, Bahador A. Can Antimicrobial Photodynamic Therapy (aPDT) Enhance the Endodontic Treatment? Journal of lasers in medical sciences 2016;7(2): 76-85.

13. Ch'ng JH, Chong KKL, Lam LN, Wong JJ, Kline KA. Biofilm-associated infection by enterococci. Nat Rev Microbiol 2019;17(2):82-94.

14. Bitoun JP, Wen ZT. Transcription factor Rex in regulation of pathophysiology in oral pathogens. Mol Oral Microbiol 2016;31(2):115-24.

15. Alghamdi F, Shakir M. The Influence of Enterococcus faecalis as a Dental Root Canal Pathogen on Endodontic Treatment: A Systematic Review. Cureus 12(3):e7257.

16. Kao PHN, Kline KA. Dr. Jekyll and Mr. Hide: How Enterococcus faecalis Subverts the Host Immune Response to Cause Infection. J Mol Biol 2019;431(16):2932-2945.

17. Prada I, Micó-Muñoz P, Giner-Lluesma T, MicóMartínez P, Collado-Castellano N, Manzano-Saiz A. Influence of microbiology on endodontic failure. Literature review. Med Oral Patol Oral Cir Bucal 2019;24(3):e364-e372.

18. Ghorbanzadeh A, Bahador A, Sarraf P, Ayar R, Fekrazad $\mathrm{R}$, Asefi S. Ex vivo comparison of antibacterial efficacy of conventional chemomechanical debridement alone and in combination with light-activated disinfection and laser irradiation against Enterococcus faecalis biofilm. Photodiagnosis Photodyn Ther 2020;29:101648.

19. Siqueira JF Jr, Rôças IN, Ricucci D, Hülsmann M. Causes and management of post-treatment apical periodontitis. Br Dent J 2014;216(6):305-12.

20. Stuart CH, Schwartz SA, Beeson TJ, Owatz CB. Enterococcus faecalis: its role in root canal treatment failure and current concepts in retreatment. J Endod 2006;32(2):93-8.

21. Vander Haar EL, So J, Gyamfi-Bannerman C, Han YW. Fusobacterium nucleatum and adverse pregnancy outcomes: Epidemiological and mechanistic evidence. Anaerobe 2018;50:55-59.

22. Prada I, Micó-Muñoz P, Giner-Lluesma T, MicóMartínez P, Collado-Castellano N, Manzano-Saiz A. Influence of microbiology on endodontic failure. Literature review. Med Oral Patol Oral Cir Bucal 2019;24(3):e364-e372

23. Chew J, Zilm PS, Fuss JM, Gully NJ. A proteomic investigation of Fusobacterium nucleatum alkalineinduced biofilms. BMC Microbiol 2012;12:189.

24. Saito Y, Fujii R, Nakagawa KI, Kuramitsu HK, Okuda K, Ishihara K. Stimulation of Fusobacterium nucleatum biofilm formation by Porphyromonas gingivalis. Oral Microbiol Immunol 2008;23(1):1-6.

25. De Andrade KQ, Almeida-da-Silva CLC, Coutinho-Silva R. Immunological Pathways Triggered by Porphyromonas gingivalis and Fusobacterium nucleatum: Therapeutic Possibilities? Mediators Inflamm 2019, 7241312.

26. Yiping W. Han. Fusobacterium nucleatum: a commensal-turned pathogen. Curr Opin Microbiol. 2015;0:141-147.

27. Beltz RE, Torabinejad M, Pouresmail M. Quantitative analysis of the solubilizing action of MTAD, sodium hypochlorite, and EDTA on bovine pulp and dentin. J Endod 2003;29:334-7.

28. Ganesh A, Veronica AK, Ashok R, Varadan P, 
Deivanayagam K. Quantification of Fusobacterium nucleatum at Depths of Root Dentinal Tubules in the Tooth Using Real-time Polymerase Chain Reaction: An in vitro Study. Cureus 2019;11(5):e4711.

29. Basrani BR, Manek S, Mathers D, Fillery E, Sodhi RN. Determination of 4- chloroaniline and its derivatives formed in the interaction of sodium hypochlorite and chlorhexidine by using gas chromatography. J Endod 2010;36:312-4.

30. Pattanshetty S, Kotrashetti VS, Bhat K, Nayak RS, Somannavar P, Pujar M. Multiplex polymerase chain reaction detection of selected bacterial species from symptomatic and asymptomatic non-vital teeth with primary endodontic infections. J Investig Clin Dent 2018;9(2):e12312.

31. Tatikonda A, Sudheep N, Biswas KP, Gowtham K, Pujari S, Singh P. Evaluation of Bacteriological Profile in the Apical Root Segment of the Patients with Primary Apical Periodontitis. J Contemp Dent Pract 2017;18(1):44-48

32. Chow AT, Quah SY, Bergenholtz G, Lim KC, Yu VSH, Tan KS. Bacterial species associated with persistent apical periodontitis exert differential effects on osteogenic differentiation. Int Endod J 2019;52(2):201210.

33. Hirota K, Yumoto H, Sapaar B, Matsuo T, Ichikawa T, Miyake Y. Pathogenic factors in Candida biofilm-related infectious diseases. J Appl Microbiol 2017;122(2):321330.

34. Siqueira JF Jr, Sen BH. Fungi in endodontic infections. Oral Surg Oral Med Oral Pathol Oral Radiol Endod 2004;97:632-41.

35. Guo D, Yue H, Wei Y, Huang G. [Genetic regulatory mechanisms of Candida albicans biofilm formation]. Sheng Wu Gong Cheng Xue Bao 2017;33(9):1567-1581.

36. Kean R, Delaney C, Rajendran R, Sherry L, Metcalfe R, Thomas R, et al. Gaining insights from Candida biofilm heterogeneity: one size does not fit all. J Fungi (Basel) 2018;4(1):12.

37. Alshanta OA, Shaban S, Nile CJ, McLean W, Ramage G. Candida albicans Biofilm Heterogeneity and Tolerance of Clinical Isolates: Implications for Secondary Endodontic Infections. Antibiotics (Basel) 2019;8(4):204.

38. Kao PHN, Kline KA. Dr. Jekyll and Mr. Hide: How Enterococcus faecalis Subverts the Host Immune Response to Cause Infection. J Mol Biol 2019;431(16):2932-2945.

39. Gulati M, Nobile CJ. Candida albicans biofilms: development, regulation, and molecular mechanisms. Microbes Infect 2016;18(5):310-21.

40. Swidergall M, Ernst AM, Ernst JF. Candida albicans mucin $\mathrm{Msb} 2$ is a broad-range protectant against antimicrobial peptides. Antimicrob Agents Chemother 2013;57(8):3917-22.

41. Gomes CC, Guimarães LS, Pinto LCC, Camargo GADCG, Valente MIB, Sarquis MIM. Investigations of the prevalence and virulence of Candida albicans in periodontal and endodontic lesions in diabetic and normoglycemic patients. J Appl Oral Sci 2017;(3):274281.

42. Yoo YJ, Kim AR, Perinpanayagam H, Han SH, Kum KY. Candida albicans Virulence Factors and Pathogenicity for Endodontic Infections. Microorganisms 2020;8(9):E1300.

43. Sangalli J, Júnior EGJ, Bueno CRE, Jacinto RC, SivieriAraújo G, Filho JEG, et al. Antimicrobial activity of
Psidium cattleianum associated with calcium hydroxide against Enterococcus faecalis and Candida albicans: anin vitro study. Clin Oral Investig 2018;22(6):2273-2279.

44. De la Torre-Luna R, Domínguez-Pérez RA, GuillénNepita AL, Ayala-Herrera JL, Martínez-Martínez RE, Romero-Ayala ME, et al. Prevalence of Candida albicans in primary endodontic infections associated with a higher frequency of apical periodontitis in type two diabetes mellitus patients. Eur J Clin Microbiol Infect Dis 2020;39(1):131-138.

45. Zhong S, Naqvi A, Bair E, Nares S, Khan AA. Viral MicroRNAs Identified in Human Dental Pulp. J Endod 2017;43(1):84-89.

46. Thompson MP, Kurzrock R. Epstein-Barr virus and cancer. Clin Cancer Res 2004;10(3):803-21.

47. Pagano JS, Whitehurst CB, Andrei G. Antiviral Drugs for EBV. Cancers (Basel) 2018;10(6):197.

48. Hernández Vigueras S, Donoso Zúñiga M, Jané-Salas E, Salazar Navarrete L, Segura-Egea JJ, Velasco-Ortega E, et al. Viruses in pulp and periapical inflammation: A review. Odontology 2016;104(2):184-91.

49. Slots J. Oral viral infections of adults. Periodontol 2000. 2009;49:60-86.

50. Jakovljevic A, Kuzmanovic Pficer J, Dragan IF, Knezevic A, Miletic M, Beljic-Ivanovic K, et al. The Role of Varicella Zoster Virus in the Development of Periapical Pathoses and Root Resorption: A Systematic Review. J Endod 2017;43(8):1230-1236.

51. Rahal EA, Hajjar H, Rajeh M, Yamout B, Abdelnoor AM. Epstein-Barr Virus and Human herpes virus 6 Type A DNA Enhance IL-17 Production in Mice. Viral Immunol 2015;28(5):297-302.

52. Ressing ME, van Gent M, Gram AM, Hooykaas MJ, Piersma SJ, Wiertz EJ. Immune Evasion by Epstein-Barr Virus. Curr Top Microbiol Immunol 2015;391:355-8.

53. Sabeti M, Valles Y, Nowzari H, Simon JH, KermaniArab V, Slots J. Cytomegalovirus and Epstein-Barr virus DNA transcription in endodontic symptomatic lesions. Oral Microbiol Immunol 2003;18(2):104-8.

54. Jakovljevic A, Nikolic N, Carkic J, Andric M, Miletic M, Beljic-Ivanovic K, et al. Notch - a possible mediator between Epstein-Barr virus infection and bone resorption in apical periodontitis. Acta Odontol Scand 2020;78(2):126-13.

55. Himi K, Takeichi O, Imai K, Hatori K, T Tamura, B Ogiso. Epstein-Barr virus reactivation by persistent apical periodontal pathogens. Int Endod J 2020;53(4):492-505. 